\title{
О КОРНЯХ УРАВНЕНИЯ ЛЭМБА ДЛЯ ДЕФОРМАЦИИ ПЛИТЫ, АНТИСИММЕТРИЧНОЙ ОТНОСИТЕЛЬНО СРЕДИННОЙ ПОВЕРХНОСТИ
}

\author{
у. нигул, \\ кандидат технических наук
}

Излагаются результаты численного и качественного исследования корней уравнення Лэмба ['], выполненного как подготовительный этап применения трехмерной теорни упругости в задачах распространения волн в упругих плитах.

Приводится диаграмма, определяющая 8 первых пар корней (корни одной пары отличаются только по знаку) уравнения Лэмба как функции частоты, а также диаграмма групповых скоростей, соответствующих чисто мнимым ветвям корней. Изучаются качественные свойства корней и рассматриваются 4 группы методов их аппроксимации: а) приближение первых корней формулами двумерных теорий, б) асимптотнческое приближение комплексных корней формулами статики при малых частотах, в) приближение малых корней при ненулевых значениях частот, г) приближение большнх мнимых корней при больших частотах.

Точные корни определены при помощи итерационного процесса, программированного А. Ю. Мяннил, которой автор выражает свою благодарность.

Расчеты были выполнены при помощи вычислительной машины М-3. Нулевые прнближения были подобраны при помоши вышеупомянутых приближенных методов.

\section{1. Диаграмма корней уравнения Лэмба}

Определим безразмерную частоту $\Omega$ по формуле

$$
\Omega=\frac{h \omega}{c_{2}},
$$

где $\omega-$ круговая частота, $h-$ полутолщина плиты, $c_{2}-$ скорость распространения волн сдвига.

Будем рассматривать $\Omega$ как вещественный параметр и примем уравнение Лэмба в форме $\left[{ }^{2}\right]$ :

$$
\begin{aligned}
& \text { где * }_{\Omega^{2}}\left(\cos \alpha \frac{\sin }{\beta}-\cos \beta \frac{\sin }{\alpha}\right)+\frac{2}{2} \cos \beta \frac{\sin }{\alpha^{2}=q^{2}+k_{0}{ }^{2} \Omega^{2},} \quad \beta^{2}=q^{2}+\Omega^{2}, \quad k_{0}^{2}=\frac{1-2 \mu}{2-2 \mu},
\end{aligned}
$$

$\mu$ - коэффициент Пуассона.

В численных расчетах данной статьи принято $\mu=0,3$.

При любом конечном значении $\Omega$ уравнение (1.2) имеет бесконечное число комплексных корней $q= \pm(\delta \pm i \vartheta)$, конечное число вещест-

* Обозначения совпадают с таковыми в $\left.{ }^{2}\right]$, если полагать $s=i \Omega$. 
венных корней $q= \pm \lambda$ и конечное число мнимых корней $q= \pm i x$. (Здесь $\delta, \vartheta, \lambda, x-$ положительные вещественные величины, зависящие от $\Omega$.

Численное исследование мнимых корней уравнения (1.2) было начато уже Лэмбом [3]. В настоящее время имеется ряд работ, указанных в $\left[{ }^{4}\right]$, которые посвящены анализу мнимых и вешественных ветвей корней уравнения (1.2). Некоторая информация о комплексных қорнях изложена в работе [5].

На рис. 1 приведена диаграмма сводного типа для $\delta_{i}, \vartheta_{j}, \lambda_{j}(j=1,2,3)$ и для $x_{k}(k=1,2, \ldots, 5)$. Она позволяет определить 8 первых пар корней $\pm q_{j}(j=1,2, \ldots, 8)$ как функции от $\Omega$.

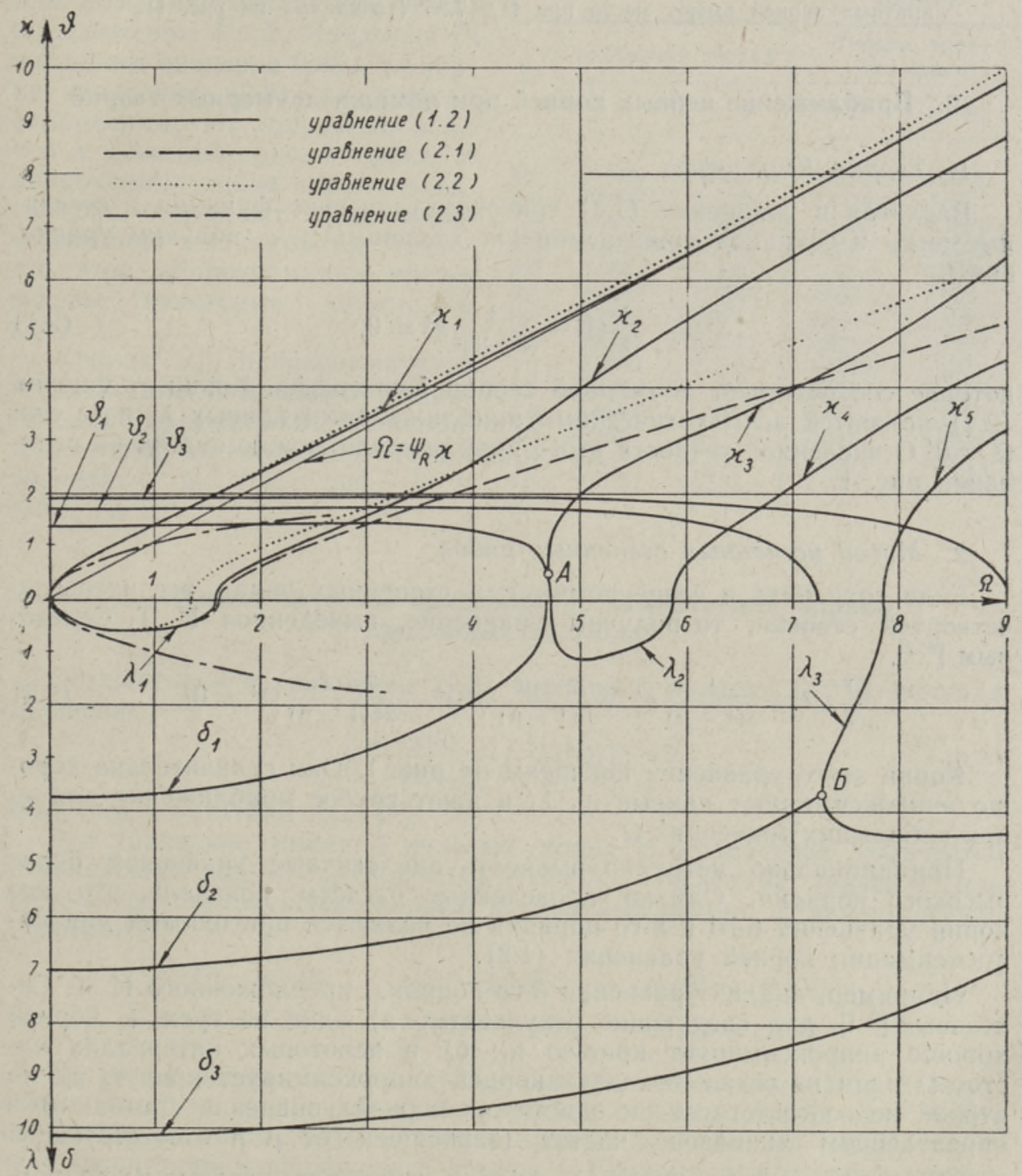

Рис. 1. Корни уравнения Лэмба (1.2) и приближенных уравнений $(2.1),(2.2),(2.3)$.

$\left(\lambda_{j}, x_{j}, \delta_{j}, \theta_{j}\right.$ обозначают соответственно абсолютные величины вещественных корней, мннмых корней, вещественной части комплексных корней и мнимой части комплексных корней). 
Из рис. 1 следует, что существуют кратные корни (точки разветвления корней) 5-ти видов:

1. Четырехкратный нулевой корень в точке $\Omega=0$.

2. Двукратные нулевые корни в точках $\Omega=\frac{m \pi}{2}$

$(m=1,3,5, \ldots \sim)$

3. Двукратные нулевые корни в точках $\Omega=\frac{n \pi}{k_{0}}$

$(n=1,2,3, \ldots \sim)$.

4. Двукратные мнимые корни $(q=i \varkappa$ и $q=-i x)$. Первые пары таких кратных корней нмеют место при $\Omega=\frac{3}{2} \pi-0,0042$ (точка «A» на рис. 1 и 2 ).

5. Двукрагые вещественные корни $(q=\lambda$ и $q=-\lambda)$. Первые пары такнх кратных корней имеют место при $\Omega=7,25$ (точка «Б» на рнс. 1).

\section{2. Приближение первых корней при помощи двумерных теорий}

\section{1. Теория Кирхгоффа}

Разлагая в уравнении (1.2) тригонометрические функции в степенные ряды и сохраняя лишь наинизкие степени $\Omega$ и $q$, получим уравнение $\left[{ }^{2}\right]$

$$
\frac{1}{2} \Omega^{2}-\frac{1}{3-3 \mu} q^{4}=0,
$$

которое соответствует двумерной теории Кирхгоффа. Корни уравнения (2.1) являются асимптотическими приближениями кривых $\lambda_{1}$ и $\varkappa_{1}$ при $\Omega \rightarrow 0$. О численной точности этого приближения можно судить на основании рис. 1.

\section{2. Метод усеченных степенных рядов}

Если сохранить в вышеупомянутых степенных рядах все члены до четвертой степени, то получим уравнение, выведенное И. Т. Селезовым $[6,7]$,

$$
\frac{1}{2} \Omega^{2}-\frac{1}{3(1-\mu)} q^{4}-\frac{2-\mu}{3(1-\mu)} q^{2} \Omega^{2}-\frac{7-8 \mu}{24(1-\mu)} \Omega^{4}=0 .
$$

Корни этого уравнения показаны на рис. 1. Они сравнительно хорошо аппроксимируют кривые $x_{1}, \lambda_{1}$ и дают грубое приближение для $\varkappa_{2}$ при небольших значениях $\Omega$.

Принципиально нетрудно выписать аналогичные уравнения более высокого порядка. Однако проведенные расчеты показали, что все корни уравнений 6-го и 8-го порядка не являются пригодными для аппроксимации корней уравнения (1.2).

Например, анализ уравнения 6-го порядка, предложенного И. Т. Селезовым $\left[{ }^{6,7}\right]$, дал следующие результаты: а) один из трех \pm корней хорошо аппроксимирует кривую $\varkappa_{1}$; б) в некоторых интервалах частот $\Omega$ один из остальных двух корней аппроксимирует кривую $x_{2}$, но второй не соответствует ни одному из корней уравнения Лэмба; в) в определенном диапазоне частот (завнсящей от $\mu$ и отсутствующей только при $\mu=\frac{1}{2}$ ) имеют место комплексные корни, которые не аппроксимируют корней уравнения Лэмба.

Была также установлена непригодность корней уравнения 8-го порядка для аппроксимацин кривых $\delta_{1}, \theta_{1}$. 
В связи с укаванным следует признать практичееки эффективным только уравнение (2.2) метода усеченных степенных рядов, если имеют в виду применение всех корней приближенного уравнения.

\section{3. Теория типа Тимошенко}

В двумерной теории типа Тимошенко [8,9] соответствует уравнению Лэмба (1.2) приближенное уравнение

$$
\frac{1}{2} \Omega^{2}-\frac{1}{3(1-\mu)}\left(q^{2}+\frac{1-\mu}{2} \Omega^{2}\right)\left(q^{2}+\frac{1}{k^{2}} \Omega^{2}\right)=0 .
$$

Если подобрать (при $\mu=0,3$ ) для коэффициента сдвига $k^{2}$ по предложению Р. Д. Миндлина [9] численное значение 0,860 , то корни уравнения (2.3) так хорошо аппроксимируют кривые $x_{1}$ и $\lambda_{1}$, что в масштабе рис. 1 точные и аппроксимирующие кривые практически совпадают. Для количественной иллюстрации точности этой аппроксимации привеведены некоторые -данные в табл. 1.

Кривая $x_{2}$ аппроксимируется теорией типа Тимошенко с практически приемлемой. точностью только при сравнительно малых значениях $\Omega$ (см. рис. 1 и табл. 1).

\begin{tabular}{|c|c|c|c|c|}
\hline \multirow{2}{*}{$\Omega$} & \multicolumn{2}{|c|}{ Трехмерная теория } & \multicolumn{2}{|c|}{$\begin{array}{r}\text { Теория типа } \\
\text { Тимошенко } \\
\end{array}$} \\
\hline & $\pm q_{1}$ & $\pm q_{2}$ & $\pm q_{1}$ & $\pm q_{2}$ \\
\hline $\begin{array}{l}0,0 \\
0,5 \\
1,0 \\
1,5 \\
2,0 \\
2,5 \\
3,0 \\
3,5 \\
4,0 \\
5,0 \\
6,0 \\
7,0 \\
8,0 \\
9,0\end{array}$ & $\begin{array}{l}0,0000 \\
0,8408 i \\
1,355 i \\
1,852 i \\
2,350 i \\
2,854 i \\
3,362 i \\
3,875 i \\
4,393 i \\
5,439 i \\
6,497 i \\
7,562 i \\
8,634 i \\
9,708 i\end{array}$ & $\begin{array}{l}0,0 w 0 \\
0,5773 \\
0,5882 \\
0,2501 \\
0,7007 i \\
1,150 i \\
1,574 i \\
2,024 i \\
2,558 i \\
3,223 i \\
5,275 i \\
6,471 i \\
7,594 i \\
8,676 i\end{array}$ & $\begin{array}{l}0,0000 \\
0,8435 i \\
1,363 i \\
1,868 i \\
2,375 i \\
2,887 i \\
3,404 i \\
3,924 i \\
4,448 i \\
5,503 i \\
6,565 i \\
7,631 i \\
8,699 i \\
9,770 i\end{array}$ & $\begin{array}{l}0,0000 \\
0,5773 \\
0,5882 \\
0,2942 \\
0,6401 i \\
1,058 i \\
1,425 i \\
1,769 i \\
2,101 i \\
2,744 i \\
3,371 i \\
3,987 i \\
4,598 i \\
5,204 i\end{array}$ \\
\hline
\end{tabular}

\section{3. Асимптотическое приближение комплексных корней при малых частотах}

Осуществляя в уравнении (1.2) переход к пределу $\Omega \rightarrow 0$, получим уравнение $[2,5]$

$$
\left[\frac{\sin 2 q(0)}{2 q(0)}-1\right][q(0)]^{2}=0
$$

известное из статики плит $\left[{ }^{10}\right]$.

Это уравнение имеет 4 нулевых корня и бесконечное количество комплексных корней $q_{j}(0)=\delta_{j}(0)+i \vartheta_{j}(0)$. Численные значения для первых трех комплексных корней, показанных на рис. 1, являются следующими [10]:

$$
\begin{array}{lll}
\delta_{1}(0)=3,749, & \delta_{2}(0)=6,950, & \delta_{3}(0)=10,119, \\
\vartheta_{1}(0)=1,384, & \vartheta_{2}(0)=1,676, & \vartheta_{3}(0)=1,858 .
\end{array}
$$

Из рис. 1 видно, что при малых значениях $\Omega$ величины $\delta_{j}$, $\hat{\vartheta}_{j}$ мало изменяются по сравнению с их асимптотическими значениями $\delta_{j}(0)$, $\vartheta_{i}(0)$, причем почти прямые участки кривых $\delta_{j}, \vartheta_{j}$ быстро увеличиваются с возрастанием порядкового номера $j$. Поэтому при малых частотах комплексные корни уравнения Лэмба (1.2) могут быть аппроксимированы комплексными корнями уравнения (3.1).

Известно, что в задачах статики с комплексными корнями уравнения (3.1) связано напряженное состояние типа эффекта Сен-Венана. 


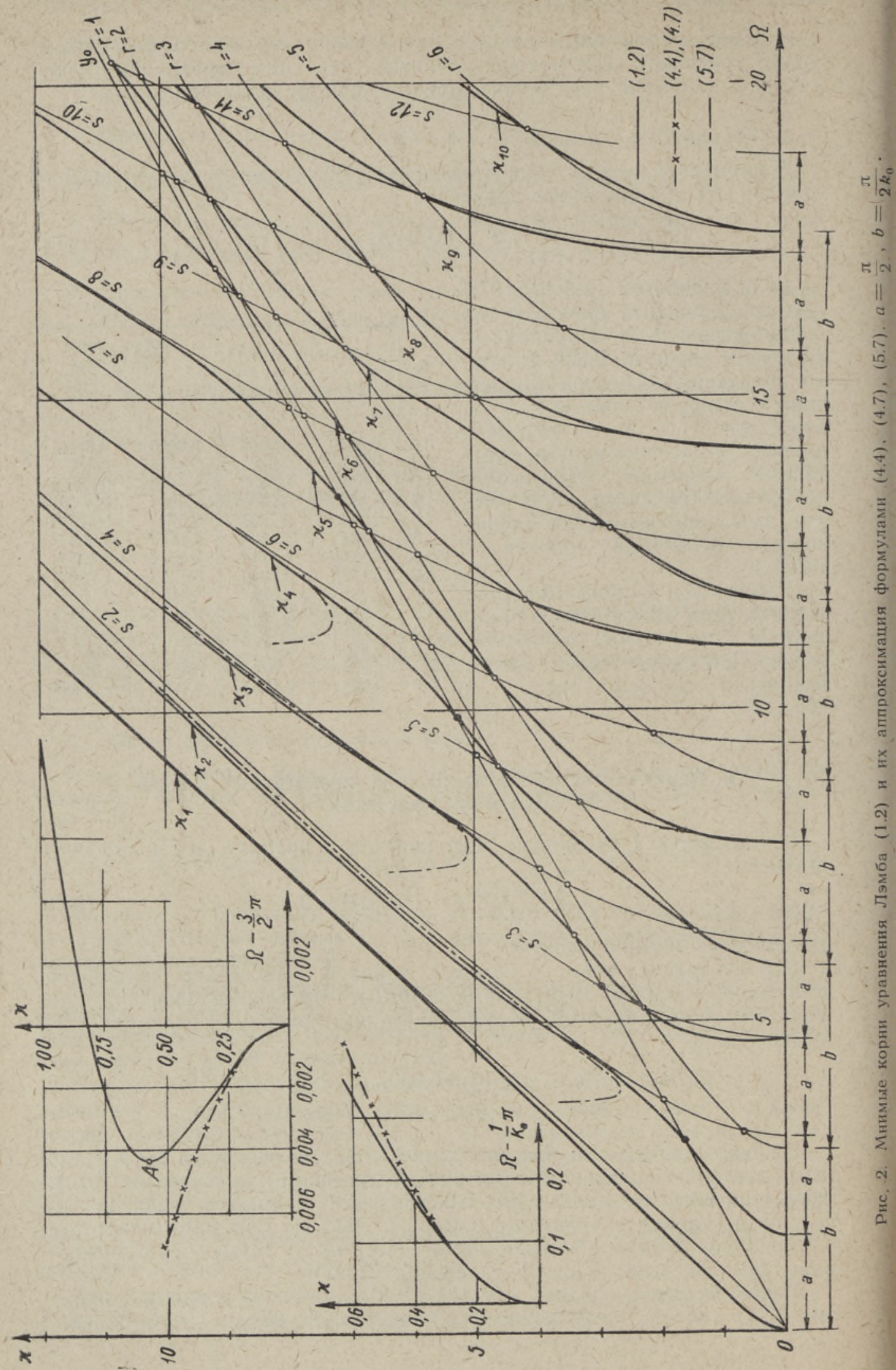


Из вышеизложенного следует, что при $\Omega \ll \frac{3}{2} \pi$ это напряженное состояние мало зависит от частоты.

\section{4. Приближение малых корней при ненулевых значениях $\Omega$}

Для приближения корней $|q| \rightarrow 0$ при $\Omega \rightarrow \frac{m \pi}{2} \quad(m=1,3,5, \ldots)$ полагаем

$$
\Omega_{m^{2}} \approx \frac{m^{2} \pi^{2}}{4}-q_{m^{2}} R_{m}
$$
мулу

Из (1.2) получим в первом приближении для постоянной $R_{m}$ фор-

$$
R_{m}=\frac{32 \alpha_{m}}{m^{2} \pi^{2} \operatorname{tg} \alpha_{m}}
$$

где

$$
\alpha_{m}=\frac{m \pi k_{0}}{2}
$$

Задавая конкретные значения $m$, легко вычислить на основе (4.2), (4.3) величины $R_{m}$. В случае $\mu=0,3$, например, имеем $R_{1}=3,44$, $R_{2}=-0,26, R_{3}=1,31$ и т. д.

Для корней $q_{m}$ получим из (4.1) формулу

$$
q_{m^{2}}=\frac{\frac{m^{2} \pi^{2}}{4}-\Omega_{m}^{2}}{R_{m}} .
$$

При положительных $R_{m}$. мнимые корни $q_{m}= \pm i \varkappa_{m}$ имеют место при $\Omega_{m}>\frac{m \pi}{2}$ и вещественные корни $q_{m}= \pm \lambda_{m}-$ при $\Omega<\frac{m \pi}{2}$, а при отрицательных $R_{m}$ - противоположно.

Выяснение указанного качественного свойства мнимых и вещественных корней представляет большой интерес, поскольку оно тесно связано с определением точек разветвления типа «A» (см. рис. 1,2$)$ и построением диаграмм отрицательных групповых скоростей (см. п. 7).

О численной точности изложенных формул можно судить на основе диаграмм, приведенных в верхней части рис. 2.

Совершенно аналогично можно построить формулы для корней $|q| \rightarrow 0$ при $\Omega \rightarrow \frac{n \pi}{k_{0}}(n=1,2,3, \ldots)$.

\section{Полагая}

$$
\Omega_{n}^{2} \approx \frac{n^{2} \pi^{2}}{k_{0}^{2}}-q_{n}^{2} S_{n},
$$

получим на основе (1.2) приближенные формулы

$$
\begin{gathered}
S_{n}=8 \frac{\operatorname{tg} \beta_{n}}{\beta_{n}}+\frac{1}{k_{0}^{2}}, \quad \beta_{n}=\frac{n \pi}{k_{0}}, \\
q_{n}^{2}=\frac{\frac{n^{2} \pi^{2}}{k_{0}^{2}}-\Omega_{n}^{2}}{S_{n}}
\end{gathered}
$$

5 ENSV TA Toimetised T-3 1963 
При положительных $S_{n}$ мнимые корни имеют место при $\Omega_{n}>\frac{n \pi}{k_{0}}$ и вещественные корни - при $\Omega_{n}<\frac{n \pi}{k_{0}}$, а при отрицательных $S_{n}$ - противоположно.

\section{5. Гриближение больших мнимых корней при больших частотах}

При $\Omega \rightarrow \sim$ кривая $x_{1}$ асимптотически приближается к прямому $\Omega=\chi \psi_{R}$, а кривые $x_{j}(j \geqslant 2)-$ к прямому $\Omega=\varkappa$. Здесь $\psi_{R}$ обозначает фазовую скорость волн Релея (при $\mu=0,3$ имеем $\psi_{R}=0,9274$ ).

Из рис. 2 следует, что кривые $x_{j}(j \geqslant 3)$ приближаются к прямому $\Omega=\varkappa$ лишь при весьма больших значениях $\Omega$.

Построим приближенные формулы, аппроксимирующие кривые $x_{j}(j \geqslant 2)$ в области $x>k_{0} \Omega$.

Заметим, что в этой области

где величины

$$
\alpha=i \alpha_{*}, \quad \beta=\beta_{*},
$$

$$
\alpha_{*}=\sqrt{\varkappa^{2}-k_{0}^{2} \Omega^{2},} \quad \beta_{*}=\sqrt{\Omega^{2}-x^{2}}
$$

являются вещественными. Предполагая, что $\alpha * 1$, принимаем

$$
\cos \alpha \approx \frac{1}{2} e^{\alpha_{*}}, \quad \frac{\sin \alpha}{\alpha} \approx \frac{1}{2 \alpha_{*}} e^{\alpha_{*}} \text {. }
$$

На основе (5.3) получим из (1.2) формулу

$$
\beta_{*} \operatorname{tg} \beta_{*} \approx-\frac{\left(x^{2}-\frac{1}{2} \Omega^{2}\right)^{2}}{x^{2} \alpha_{*}} .
$$

Предполагая далее, что

$$
\boldsymbol{\beta}_{*}=n \pi+T, \quad T=T(\Omega) \ll n \pi \quad(n=1,2, \ldots),
$$

получим из (5.4) в первом приближении формулу

$$
\operatorname{tg} T \approx-\frac{\left(\frac{1}{2} \Omega^{2}-n^{2} \pi^{2}\right)^{2}}{n \pi\left(\Omega^{2}-n^{2} \pi^{2}\right) \sqrt{\left(1-k_{0}^{2}\right) \Omega^{2}-n^{2} \pi^{2}}} .
$$

При численно заданных $n$ и $\Omega$ формулы (5.5), (5.6) позволяют сравнительно просто определить приближенные значения $\approx$ по формуле

$$
x \approx \sqrt{\beta_{*}^{2}-\Omega^{2}}
$$

Результаты, представленные на рис. 2, позволяют признать такое приближение практически приемлемым при достаточно больших значениях $\Omega$ и $x$.

\section{6. «Oпорные точки» диаграмм мнимых корней}

Кривые $x_{j}(j \geqslant 2)$ пересекают прямую $y_{0}=k_{0} \Omega$ в точках, где

$$
\begin{aligned}
& \alpha=0, \quad \cos \alpha=\frac{\sin \alpha}{\alpha}=1, \\
& \beta=\frac{\Omega}{\sqrt{2-2 \mu}}=\frac{x}{\sqrt{1-2 \mu}} .
\end{aligned}
$$


Для определения этих точек получим из (1.2) уравнение

$$
\frac{\operatorname{tg} \beta}{\beta}=-\frac{\mu^{2}}{1-2 \mu} \text {. }
$$

При $\mu=0,3$ первые корни уравнения (6.3) имеют значения: $\beta_{1}=2,61$, $\beta_{2}=5,40, \beta_{3}=8,34, \beta_{4}=11,37$.

На. рис. 1 корням уравнения (6.3) соответствуют черные точки.

При конструировании кривых $x_{j}(j \geqslant 3)$ в области $k_{0} \Omega>x_{j}>0$ целесообразно пользоваться еще «опорными точками» вспомогательной сетки Р. Д. Миндлина [ $\left.{ }^{4}\right]$. Эта, сетка показана на рис. 2 тонкими линиями, а «опорные точки» - малыми кружочками. ниями

Одна группа кривых вспомогательной сетки определена уравне-

$$
y_{r}=\sqrt{k_{0}^{2} \Omega^{2}-\frac{r^{2} \pi^{2}}{4}} \quad(r=1,2,3, \ldots)
$$

и вторая группа - уравнениями

$$
z_{s}=\sqrt{\Omega^{2}-\frac{s^{2} \pi^{2}}{4}} \quad(s=1,2,3, \ldots) .
$$

В точках пересечения этих кривых

$$
y_{r}=z_{s}, \quad \Omega=\frac{\pi}{2} \frac{\sqrt{s^{2}-r^{2}}}{\sqrt{1-k_{0}^{2}}} .
$$

Легко убедиться в том, что те из точек (6.6), где $r$ и $s$ одновременно имеют либо четные, либо нечетные значения, являются решениями уравнения Лэмба (1.2). Поскольку эти точки легко вычисляются, их можно рассматривать как «опорные точки» диаграмм $x_{j}(j \geqslant 3)$.

\section{7. Диаграммы групповых скоростей}

При решении конкретных задач играют важную роль диаграммы групповых скоростей, соответствующие мнимым корням уравнения Лэмба [1']. Итак, рассмотрим случай

$$
q=i x, \quad \alpha=\sqrt{k_{0}^{2} \Omega^{2}-x^{2}}, \quad \beta=\sqrt{\Omega^{2}-x^{2}} .
$$

На основе (1.2) получим для групповой скорости $\frac{d \Omega}{d x}$ формулу:

где

$$
\frac{d \Omega}{d \varkappa}=\frac{\varkappa}{\Omega} \frac{\varphi_{1}}{\varphi_{2}},
$$

$$
\begin{aligned}
& \varphi_{1}=\left(\Omega^{2}-2 x^{2}\right) \frac{\sin \alpha}{\alpha}\left[\frac{1}{4}\left(\Omega^{2}-2 x^{2}\right) \frac{\sin \underline{\beta}}{\beta}-2 \cos \beta\right]+ \\
& +\frac{1}{4}\left(\Omega^{2}-2 \chi^{2}\right)^{2} \frac{\cos \beta}{\alpha^{2}}\left[-\frac{\sin \alpha}{\alpha}-\cos \alpha\right]+ \\
& +\frac{\sin \beta}{\beta}\left[x^{2} \beta^{2} \frac{\sin \alpha}{\alpha}+\left(2 \Omega^{2}-3 x^{2}\right) \cos \alpha\right]-x^{2} \cos \alpha \cos \beta \text {, } \\
& \varphi_{2}=\left(\Omega^{2}-2 \chi^{2}\right) \frac{\sin \alpha}{\alpha}\left[\frac{1}{4}\left(\Omega^{2}-2 \varkappa^{2}\right) \frac{\sin \beta}{\beta}-\cos \beta\right]+ \\
& +\frac{k_{0}^{2}}{4}\left(\Omega^{2}-2 \varkappa^{2}\right)^{2}-\frac{\cos 6}{\alpha^{2}}\left[\frac{\sin \alpha}{\alpha}-\cos \alpha\right]+ \\
& +x^{2} \frac{\sin \beta}{\beta}\left[k_{0}^{2} \beta^{2} \frac{\sin \alpha}{\alpha}-\cos \alpha\right]-\chi^{2} \cos \alpha \cos \beta \text {. }
\end{aligned}
$$




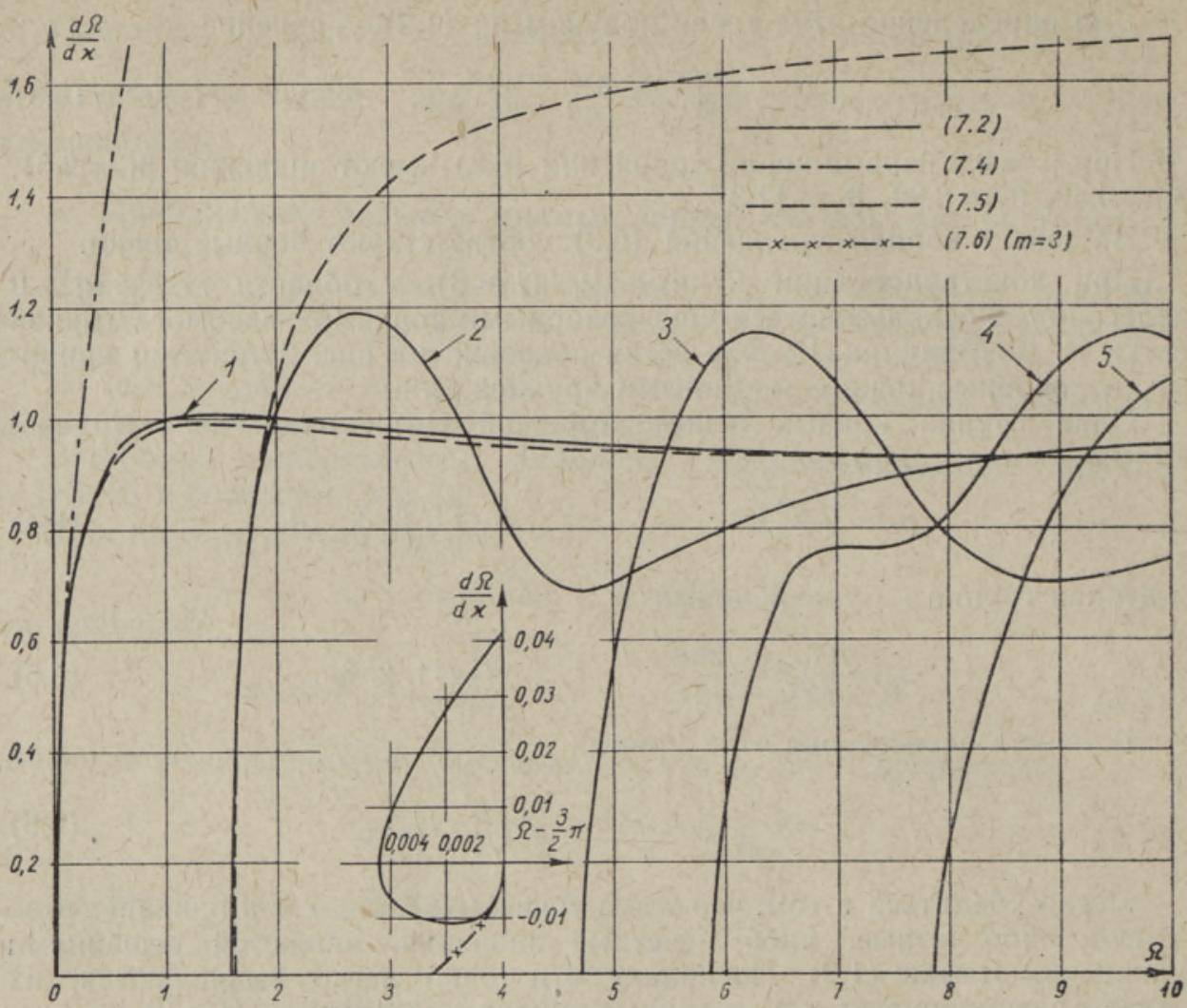

Рис. 3. Диаграммы групповых скоростей по точной формуле (7.2) и по приближенным формулам (7.4), (7.5), (7.6).

На основе теории Кирхгоффа получим приближенно

$$
\frac{d \Omega}{d x}=2 \sqrt[4]{\frac{2}{3-3 u}} \sqrt{\Omega}
$$

а на основе теории типа Тимошенко -

$$
\frac{d \Omega}{d \varkappa}=\frac{x}{\Omega} \frac{4 \varkappa^{2}-\left(1-\mu+\frac{2}{k_{0}^{2}}\right) \Omega^{2}}{3-3 \mu+\left(1-\mu+\frac{2}{k_{0}^{2}}\right) \varkappa^{2}-\frac{2-2 \mu}{k_{0}^{2}} \Omega^{2}} .
$$

Формулам (4.1), (4.5) соответствуют выражения групповой скорости

$$
\frac{d \Omega}{d x}=\frac{\varkappa}{\Omega} R_{m}, \quad \frac{d \Omega}{d \chi}=\frac{\varkappa}{\Omega} S_{n} .
$$

На рис. 3 показаны диаграммы групповых скоростей, которые соответствуют кривым $x_{j}(j=1,2, \ldots, 5)$ на рис. 1 . Одной из этих кривых $x_{j}$. именно кривой $\varkappa_{3}$, соответствуют (в диапазоне от точки $x_{3}=0$ до точки «A») отрицательные значения $\frac{d \Omega}{d x}$, которые в увеличенном масштабе отдельно показаны на рис. 3 . 
При отрицательных $R_{m}$ и $S_{n}$ групповая скорость $\frac{d \Omega}{d \%}$ и фазовая скорость $\frac{Q}{x}$ имеют противоположные знаки в окрестности точек $\Omega=\frac{1}{2} m \pi$, $\Omega=k_{0}{ }^{-1} n \pi$. Такие участки диаграмм групіповых скоростей, впервые численно исс́ледованные недавно [11], принято называть участками «отрицательных групповых скоростей», хотя более логично было бы говорить об отрицательных фазовых скоростях. Осуществляя, например, в точке А аналитическое продолжение корней, нетрудно убедиться в том, что «отрицательные» и «положительные» участки диаграммы групповых скоростей соответствуют различным корням.

Из формул (7.3), а также из сопоставления рис. 3 с диаграммами других авторов [1'] следует, что кривые $\frac{d \Omega}{d x}$ являются очень «чувствительными» относительно коэффициента Пуассона.

На рис. 3 показаны также кривые, построенные на основе формул (7.4), (7.5) двумерных теорий. Теория Кирхгоффа хорошо аппроксимирует лишь первую кривую $\frac{d \Omega}{d \varkappa}$ при очень малых значениях $\Omega$. Теория типа Тимошенко хорошо аппроксимирует первую кривую $\frac{d \Omega}{d x}$ при любых значениях $\Omega$, а вторую - только при $\Omega \leqslant 2$.

\section{ЛИТЕРАТУРА}

1. H. L a m b, On the Flexure of an Elastic Plate (Appendix), Proc. Lond. Math. Soc., vol. 21, p. $85,1889-1890$.

2. У. К. Ни гул, О применении символического метода А. И. Лурье в трехмерной теории динамики упругих плит, Изв. АН ӘССР, т. ХІІ, № 2, 1963.

3. H. L a m b, On Waves in an Elastic Plate, Proc. Roy. Soc., A, vol. 93, 1917, p. 114.

4. R. D. Mindlin, Waves and Vibrations in Isotropic, Elastic Plates, «Structural Mechanics», Perg. Press, p. 199, 1960.

5. J. W. Sherw oo d, Propagation in an Infinite Elastic Plate, J. Acoust. Soc. Amer., vol. 30 , No. 10,1958 , p. 979 .

6. I. Т. Селезов, Дослідження поперечних коливань пластини, Прикладна механіка, т. VI, в. 5,1960 , стр. 319.

7. І. Т. Селезов, Про поперечні коливання пластини, Доп. АН Укр. РСР, 9, 1960. стр. 1190.

8. Я. С. У флянд, Распространение волн при поперечных колебаниях стержней и пластин, ПММ, т. XII, вып. 3, 1948, стр. 287.

9. R. D. M ind I in, Influence of Rotatory Inertia and Shear on Flexural Motions of Isotropic Elastic Plates, J. Appl. Mech., vol. 18, No. 1, 1951, p. 31.

10. А. И. Лурье, К теории толстых плит, ПММ, т. VI, 1942, стр. 152.

11. I. Tolstoy, E. Usdin, Wave Propagation in Elastic Plates: Low and High ModeDispersion, J. Acoust. Soc. Amer., vol. 29, No. 1, 1957, p. 37.

Ннститут кибернетики

Академии наук Эстонской ССР
Поступила . в редакцию

19. X 1962 


\section{LAMBI VÕRRANDI JUURTEST PLAADI KESKPINNA SUHTES ANTISOMMEETRILISE DEFORMATSIOONI PUHUL}

\section{U. Nigul, \\ tehnikateaduste kandidaat \\ Resümee}

Esitatakse Lambi vörrandi (1.2) juurte ja grupikiiruste (7.2) uurimise tulemusi ning käsitletakse mitmesuguseid juurte aproksimeerimise meetodeid. Reaalsete $(q= \pm \lambda)$ ja imaginaarsete $(q= \pm i x)$ harude kõrval vaadeldakse ka juurte kompleksseid harusid $q= \pm(\delta \pm i \vartheta)$.

Numbrilised tulemused esitatakse joonistel 1, 2, 3 ning tabelis 1. Arvutustes kasutati Poisson'i tegurit $\mu=0,3$.

Eesti NSV Teaduste Akadeemia Küberneetika Instituut

Saabus toimetusse

19. X 1962

\section{ON THE ROOTS OF LAMB EQUATION FOR A DEFORMATION ANTISYMMETRIC ABOUT THE MIDDLE PLANE OF THE PLATE}

\section{U. Nigul \\ Sumımary}

The results of a numerical research of Lamb equation (1.2) roots and group velocities (7.2) are presented, and several methods for roots approximation are discussed. Besides real $(q= \pm \lambda)$ and imaginary $(q= \pm i x)$ branches also the complex branches $q= \pm(\delta \pm i \vartheta)$ of roots are investigated

The numerical results are given in figs. 1, 2, 3 and in table 1. In calculations Poisson's ratio $\mu=0.3$ has been used.

Academy of Sciences of the Estonian S.S.R.,

Received Institute of Cybernetics 